\title{
Identification key for lycophytes and ferns from the Picinguaba and Santa Virgínia Nuclei, Parque Estadual da Serra do Mar, Ubatuba, SP, Brazil
}

\author{
Giseli Areias Nóbrega $^{1, *}$, Marcos Pereira Marinho Aidar ${ }^{2}$, Mateus Paciencia $^{3}$ \& Jefferson Prado ${ }^{4}$ \\ ${ }^{I}$ Fundação Florestal, Jardim Botânico de Cubatão, Diretoria Geral, São Paulo, SP, Brazil \\ ${ }^{2}$ Instituto de Botânica, Núcleo de Pesquisas em Fisiologia e Bioquímica, São Paulo, SP, Brazil \\ ${ }^{3}$ Universidade Paulista, Laboratório de Extração, São Paulo, SP, Brazil \\ ${ }^{4}$ Instituto de Botânica, Núcleo de Pesquisas Curadoria do Herbário SP, São Paulo, SP, Brazil \\ *Corresponding author: Giseli Areias Nóbrega,e-mail: giareias@gmail.com
}

NÓBREGA, G.A., AIDAR, M.P.M., PACIENCIA, M., PRADO, J. Identification key for lycophytes and ferns from the Picinguaba and Santa Virgínia Nuclei, Parque Estadual da Serra do Mar, Ubatuba, SP, Brazil. Biota Neotropica. 16(4): e20150144. http://dx.doi.org/10.1590/1676-0611-BN-2015-0144

\begin{abstract}
This work contributes to the knowledge of the fern and lycophytes diversity in the Picinguaba and Santa Virginia Nuclei, Parque Estadual da Serra do Mar (PESM), Ubatuba, São Paulo, Brazil, as well as to provide subsidies to identification of the found taxa in these areas. In total, 30 collecting expeditions were conducted and the vouchers were deposited at UEC Herbarium. We have identified 135 species distributed in 53 genera and 19 families for both areas. The most representative families were Polypodiaceae (28 species) and Dryopteridaceae (19 species), whereas Asplenium (12 species), Blechnum and Elaphoglossum (8 species each) were the most important genera. Epiphytic species predominated ( 62 overall), probably due to the presence of favorable habitats. Among the taxa, 41 are considered endemic from Brazil, 35 of them endemic to the Brazilian Atlantic Forest. Five species are classified as vulnerable according to the "Official list of endangered species in the State of São Paulo": Elaphoglossum macahense (Fée) Rosenst., Anetium citrifolium (L.) Splitg., Asplenium muellerianum Rosenst., Cyathea glaziovii (Fée) Domin, and Thelypteris angustifolia (Willd.) Proctor, which emphasizes the importance of preservation areas such as the Parque Estadual da Serra do Mar. An identification key has been elaborated to help with the recognition of these groups in Picinguaba and Santa Virgínia Nuclei.
\end{abstract}

Keywords: Floristics, taxonomy, diversity, Atlantic Rain Forest

\section{Chave de identificação para licófitas e samambaias dos Núcleos Picinguaba e Santa Virgínia, Parque Estadual da Serra do Mar, Ubatuba, SP, Brasil}

\begin{abstract}
Resumo: Este trabalho visa contribuir para o conhecimento da diversidade de licófitas e samambaias ocorrentes nos núcleos Picinguaba e Santa Virgínia do Parque Estadual da Serra do Mar (PESM), Ubatuba, SP, Brasil, bem como fornecer subsídios para a identificação dos táxons encontrados nestas áreas. Ao todo, foram realizadas 30 expedições de coleta e o material botânico encontra-se depositado no Herbário UEC. Foram identificadas 135 espécies, 19 famílias e 53 gêneros para as duas áreas. As famílias mais representativas foram Polypodiaceae (28 espécies) e Dryopteridaceae (19 espécies), enquanto Asplenium (12 espécies), Blechnum e Elaphoglossum (8 espécies cada) foram os gêneros melhor representados. As espécies epífitas predominaram (62 ao todo), provavelmente devido à presença de hábitats favoráveis. Dos táxons encontrados, 41 são considerados endêmicos do Brasil, sendo 35 deles endêmicos da Mata Atlântica brasileira; cinco espécies são classificadas como vulneráveis, conforme a "Lista oficial de espécies ameaçadas de extinção no Estado de São Paulo": Elaphoglossum macahense (Fée) Rosenst., Anetium citrifolium (L.) Splitg., Asplenium muellerianum Rosenst., Cyathea glaziovii (Fée) Domin e Thelypteris angustifolia (Willd.) Proctor, o que ressalta a importância de áreas de preservação como o Parque Estadual da Serra do Mar, no contexto conservacionista. Uma chave de identificação foi elaborada para contribuir no reconhecimento destes grupos nos núcleos Picinguaba e Santa Virgínia.
\end{abstract}

Palavras-chaves: florística, taxonomia, diversidade, Floresta Ombrófila Densa Atlântica 


\section{Introduction}

The Atlantic Forest is a complex set of ecosystems, with significant importance for harboring a considerable portion of Brazilian biological diversity (Joly et. al., 1999). According to Forzza et al. (2012) this vegetation presents 19,335 species of plants and fungi, which contributes substantially to the mega-diversity of the country.

However, studies reveal that only $7.9 \%$ of the Atlantic Forest remnants are larger than 100 hectares, a size that would be representative for biodiversity conservation (SOS Atlantic Forest and INPE, 2011). These data reinforce the need to better understand and protect this forest, which is considered one of the major biodiversity hotspots of the Neotropics (Myers et al., 2000).

The Serra do Mar (a mountain range within the Atlantic Forest Domain) is located mainly in the Southeastern region of Brazil. It is a primary center of diversity and endemism for lycophytes and ferns (Tryon, 1986), probably because of regional relief features. As amply documented in several previous studies, mountainous areas have favorable environmental conditions for the development of these two groups of plants (see Holttum, 1938; Page, 1979; Tryon 1986; Roos, 1996, and Moran, 2008).

Recently, Prado et al. (2015) reported 883 species of ferns and lycophytes in the Brazilian Atlantic Rain Forest. This number surpasses Amazon (with 503 species), Cerrado (269 species), Caatinga (26 species), Pantanal (30 species), and Pampa (eight species). In the Atlantic Rain Forest of São Paulo there are 603 species, distributed in 115 genera and 33 families of ferns and lycophytes. According to Prado (1998), the highest diversity of ferns and lycophytes within this area is found at Serra da Mantiqueira, Serra da Bocaína, Serra do Japi, and Vale do Ribeira.

For similar areas to the current study (i.e., Atlantic Forest mountain slopes in the state of São Paulo), there are the works by Custódio-Filho (1989), Prado \& Labiak (2001, 2009); Athayde-Filho et al. (2003), Prado (2004), Boldrin \& Prado (2007), Salino \& Almeida (2008), and Mazziero et al. (2015). However, there are no identification keys for the ferns and lycophytes from the Atlantic Forest in northeastern São Paulo.

The Picinguaba and Santa Virgínia Nuclei are located in the Parque Estadual da Serra do Mar, municipalities of Ubatuba, São Luís do Paraitinga, and Cunha, protecting a very well preserved area of Atlantic Forest at northeastern São Paulo. These places harbor a rich flora of lycophytes and ferns and the main objective of this paper is to provide an identification key for determining the species of these groups in these areas.

\section{Material and Methods}

Study area: The "Parque Estadual da Serra do Mar" (State Park) represents the largest continuous area of well preserved Atlantic Forest in Brazil, with almost 315000 ha $\left(3.15^{9} \mathrm{~m}^{2}\right)$ and covering areas from two states, São Paulo and Rio de Janeiro, in the southeast region of the country. This park is divided into 10 conservation units, among them Picinguaba and Santa Virginia.

The Santa Virginia Nucleus (SVN) belongs to the municipalities of São Luís do Paraitinga (70\% of its area), Cunha (20\%), and Ubatuba $(10 \%)$, at coordinates $23^{\circ} 17^{\prime}-23^{\circ} 24^{\prime} \mathrm{S}$ and $45^{\circ} 03^{\prime}-45^{\circ} 11^{\prime} \mathrm{W}$, spanning about 5,000 ha $\left(5^{7} \mathrm{~m}^{2}\right)$ of predominantly Montane Dense Ombrophilous Forest (Veloso et al., 1991). Altitudes vary from 850 to $1,100 \mathrm{~m}$, with an average annual temperature of $17^{\circ} \mathrm{C}$, and average annual rainfall of 2,300 mm (Salemi, 2009).

The Picinguaba Nucleus (PN) is located in the Municipality of Ubatuba, at coordinates $23^{\circ} 31^{\prime}-23^{\circ} 34^{\prime} \mathrm{S}$ and $45^{\circ} 02^{\prime}-45^{\circ} 05^{\prime} \mathrm{W}$, and consists of about 47,500 ha $\left(4,75 \mathrm{~m}^{2}\right)$, according to SMA (1996). There are different types of vegetations in PN: Pioneer Formations with Marine Influence (Dunes); Pioneer Formations with Fluvial Influence (Caxetal); Pioneer Formations with Fluvial-Marine Influence (Mangrove); Lowland Dense Ombrophilous Forest (Restinga Forest); Submontane Dense Ombrophilous Forest; and Montane Dense Ombrophilous Forest (Assis, 1999). The altitude varies from sea level up to $1,340 \mathrm{~m}$, with an annual average temperature of $22^{\circ} \mathrm{C}$, and average annual rainfall exceeding 2,200 $\mathrm{mm}$ (Setzer 1966).

The study areas covers four different vegetation types in Atlantic Forest, designated according to Veloso et al. (1991) as: Lowland Dense Ombrophilous Forest, (Figure 1 - B, C, D, E, F), Submontane Dense Ombrophilous Forest (Figure 1 - G, H, I, J), Montane Dense Ombrophilous Forest (Figure 1 - K, L, M, N) and Alluvial Dense Ombrophilous Forest (Figure 1 - A). According to observations by Assis (1999), this last area is treated as Restinga Forest in the scope of the Biota FAPESP, Project, $\mathrm{n}^{\mathrm{o}} 03 / 12595-7$.

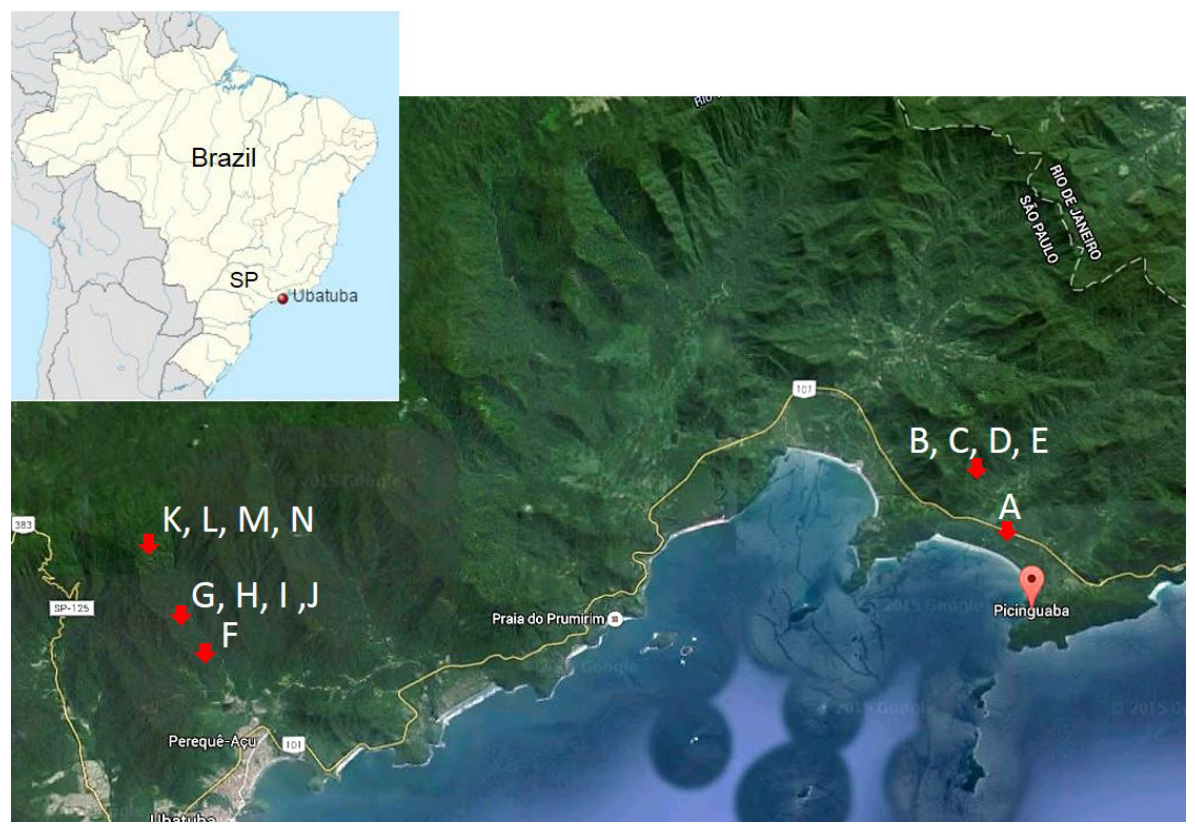

Figure 1. Location of the study areas in the Parque Estadual da Serra do Mar. A= Restinga; B, C, D, E, F= Lowlands Rain Forest; G, H, I, J= Submontane Rain Forest; K, L, M, N= Montane Rain Forest. (Google Earth in 17 Nov 2015). 
Sampling design: The lycophyte and fern of the Picinguaba and Santa Virginia Nuclei was investigated along trails, roads, and plots found in these areas. We conducted 23 collecting expeditions in the Picinguaba Nucleus, from 2008 to 2011.Eleven of these expeditions were made to the "Casa da Farinha" (Figure 1 - B, C, D, E), seven to the "Fazenda Capricórnio" (Figure 1 - F, G, H, I, J), and five to the "Praia da Fazenda" areas (Figure 1 - A). Seven expeditions with the same purpose were undertaken in Santa Virginia Nucleus (Figure 1 - K, L, M, N), totaling 30 collecting expeditions in both Nuclei.

The collected material was prepared according to the methodology indicated by Silva (1989). Herbarium vouchers were deposited at UEC. We adopted the classification system by Smith et al. (2006, 2008), with changes by Christenhusz et al. (2011).

For the geographic distribution, we adopted four basic categories: Pantropical (species of the tropical zones), Tropical America (species of the tropical and subtropical America, including South of the USA), South America (only in South America), and Endemics from Brazil. The distribution of the endemic species was based on Prado et al. (2015). The habitat and growth are reported as guilds of the life forms, according to Paciencia (2008).
The identification key here presented was prepared through the observation of the morphological features of adult plants recorded from Picinguaba and Santa Virginia Nuclei. This key prioritizes the morphological features that are easily observed in the field as well as on herbarium specimens. Therefore, the intention was to develop a key containing the maximum set of possible macroscopic features and to avoid the practice of cutting herbarium specimens.

The key does not present families or genera. We avoided presenting our results in the traditional way, because we believe that the users of this key could be enthusiasts in the study of lycophytes and ferns and they are familiarized with the common morphological features of the different groups. Unlike keys that separate different groups, the present paper pays attention to the morphological structures that are similar between species, even if they are phylogenetically distant.

\section{Results and Discussion}

We found 135 species distributed in 53 genera and 19 families of ferns and lycophytes for the two areas (Table 1). The most representative families were Polypodiaceae, with 28 species, and Dryopteridaceae, with 19 species. The genera with more species were Asplenium (12 species), followed by Blechnum and Elaphoglossum (eight species each).

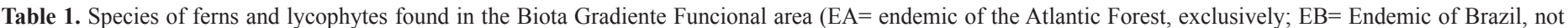

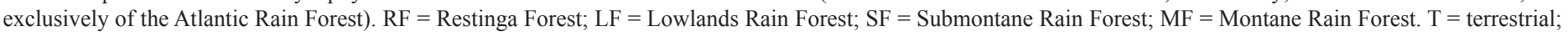
$\mathrm{R}=$ rupicolous; $\mathrm{E}=$ epiphyte; $\mathrm{H}=$ hemiepiphyte; $\mathrm{S}=$ scandent; $\mathrm{Sb}=$ subarborescent $\mathrm{A}=$ arborescent.

\begin{tabular}{|c|c|c|c|}
\hline Family & Species & Forest type & Life form \\
\hline Anemiaceae & ${ }^{E A}$ Anemia mandioccana Raddi & RF, LF & $\mathrm{FT}, \mathrm{R}, \mathrm{E}$ \\
\hline Anemiaceae & Anemia phyllitidis (L.) Sw.var. phyllitidis & $\mathrm{LF}$ & $\mathrm{T}, \mathrm{R}$ \\
\hline Aspleniaceae & Asplenium auriculatum (Hook. f.) C.V. Morton \& Lellinger & LF & $\mathrm{E}$ \\
\hline Aspleniaceae & Asplenium claussenii Hieron. & LF, SF & $\mathrm{E}$ \\
\hline Aspleniaceae & EA Asplenium kunzeanum Hieron. & $\mathrm{MF}$ & $\mathrm{T}$ \\
\hline Aspleniaceae & Asplenium mucronatum C. Presl & $\mathrm{LF}, \mathrm{SF}, \mathrm{MF}$ & $\mathrm{E}$ \\
\hline Aspleniaceae & 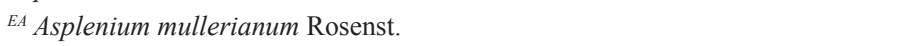 & SF & $\mathrm{E}$ \\
\hline Aspleniaceae & Asplenium oligophyllum Kaulf. & $\mathrm{SF}, \mathrm{MF}$ & $\mathrm{T}$ \\
\hline Aspleniaceae & ${ }^{E A}$ Asplenium pseudonitidum Raddi & $\mathrm{LF}, \mathrm{SF}, \mathrm{MF}$ & $\mathrm{T}$ \\
\hline Aspleniaceae & Asplenium pteropus Mett. & $\mathrm{LF}, \mathrm{SF}, \mathrm{MF}$ & $\mathrm{E}$ \\
\hline Aspleniaceae & Asplenium raddianum Gaudich. & MF & $\mathrm{E}$ \\
\hline Aspleniaceae & Asplenium radicans (Raddi) Lellinger & MF & $\mathrm{E}$ \\
\hline Aspleniaceae & Asplenium scandicinum Kaulf. & MF & $\mathrm{E}$ \\
\hline Aspleniaceae & Asplenium serratum L. & RF & $\mathrm{E}$ \\
\hline Aspleniaceae & Hymenasplenium triquetrum (N. Murak. \& R.C. Moran) L. Regalado \& C. Prada & $\mathrm{LF}, \mathrm{SF}$ & $\mathrm{T}, \mathrm{R}$ \\
\hline Athyriaceae & Diplazium cristatum (Desr.) Alston & LF, SF & $\mathrm{T}$ \\
\hline Athyriaceae & Diplazium riedelianum (Bong. ex Kuhn) Kuhn ex C. Chr. & SF & $\mathrm{T}$ \\
\hline Blechnaceae & Blechnum brasiliense Desv. & LF & $\mathrm{Sb}$ \\
\hline Blechnaceae & Blechnum acutum (Desv.) Mett. & $\mathrm{LF}, \mathrm{SF}, \mathrm{MF}$ & $\mathrm{H}$ \\
\hline Blechnaceae & Blechnum cordatum (Desv.) Hieron. & MF & $\mathrm{T}$ \\
\hline Blechnaceae & Blechnum gracile Kaulf. & LF & $\mathrm{T}, \mathrm{R}$ \\
\hline Blechnaceae & Blechnum schomburgkii (Klotzsch) C. Chr. & MF & $\mathrm{S}$ \\
\hline Blechnaceae & Blechnum lanceola Sw. & LF & $\mathrm{T}, \mathrm{R}$ \\
\hline Blechnaceae & Blechnum occidentale L. & LF & $\mathrm{T}$ \\
\hline Blechnaceae & Blechnum polypodioides Raddi & LF & $\mathrm{T}$ \\
\hline Blechnaceae & Salpichlaena volubilis (Kaulf.) J. Sm. & SF & $\mathrm{S}$ \\
\hline Cyatheaceae & Alsophila setosa (Kaulf.) Domin & MF & A \\
\hline Cyatheaceae & ${ }^{E B}$ Alsophila sternbergii (Sternb.) D.S. Conant & $\mathrm{LF}, \mathrm{SF}, \mathrm{MF}$ & A \\
\hline Cyatheaceae & Cyathea atrovirens (Langsd. \& Fisch.) Domin & $\mathrm{RF}, \mathrm{LF}, \mathrm{MF}$ & A \\
\hline Cyatheaceae & Cyathea delgadii Sternb. & $\mathrm{LF}, \mathrm{MF}$ & A \\
\hline Cyatheaceae & ${ }^{E A}$ Cyathea dichromatolepis (Fée) Domin & MF & A \\
\hline Cyatheaceae & ${ }^{E A}$ Cyathea glaziovii (Fée) Domin & SF & A \\
\hline Cyatheaceae & 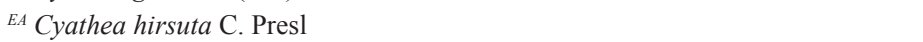 & MF & A \\
\hline
\end{tabular}


Table 1. Continued..

\begin{tabular}{|c|c|c|c|}
\hline Family & Species & Forest type & Life form \\
\hline Cyatheaceae & ${ }^{E A}$ Cyathea leucofolis Domin. & LF & A \\
\hline Cyatheaceae & ${ }^{E A}$ Cyathea phalerata Mart. & $\mathrm{LF}, \mathrm{SF}, \mathrm{MF}$ & A \\
\hline Dicksoniaceae & Lophosoria quadripinnata (J.F. Gmel.) C. Chr. & MF & $\mathrm{Sb}$ \\
\hline Dryopteridaceae & Bolbitis serratifolia (Mart. ex Kaulf.) Schott & $\mathrm{LF}, \mathrm{SF}$ & $\mathrm{R}$ \\
\hline Dryopteridaceae & ${ }^{E A}$ Ctenitis deflexa (Kaulf.) Copel. & $\mathrm{RF}, \mathrm{LF}, \mathrm{SF}, \mathrm{MF}$ & $\mathrm{T}$ \\
\hline Dryopteridaceae & Ctenitis submarginalis (Langsd. \& Fisch.) Ching & MF & $\mathrm{T}$ \\
\hline Dryopteridaceae & Didymochlaena truncatula (Sw.) J. Sm. & $\mathrm{SF}, \mathrm{MF}$ & $\mathrm{T}$ \\
\hline Dryopteridaceae & ${ }^{E A}$ Elaphoglossum chrysolepis (Fée) Alston & $\mathrm{SF}, \mathrm{LF}$ & $\mathrm{E}$ \\
\hline Dryopteridaceae & ${ }^{E A}$ Elaphoglossum lingua (C. Presl) Brack. & $\mathrm{RF}, \mathrm{LF}, \mathrm{MF}$ & $\mathrm{E}$ \\
\hline Dryopteridaceae & 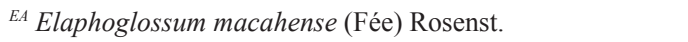 & $\mathrm{RF}$ & $\mathrm{E}$ \\
\hline Dryopteridaceae & Elaphoglossum decoratum (Kunze) T. Moore & $\mathrm{LF}, \mathrm{SF}, \mathrm{MF}$ & $\mathrm{E}$ \\
\hline Dryopteridaceae & Elaphoglossum horridulum (Kaulf.) J. Sm. & $\mathrm{LF}$ & $\mathrm{E}$ \\
\hline Dryopteridaceae & Elaphoglossum luridum (Fée) Christ & $\mathrm{LF}$ & $\mathrm{E}$ \\
\hline Dryopteridaceae & Elaphoglossum macrophyllum (Mett. ex Kuhn) Christ & $\mathrm{RF}, \mathrm{MF}$ & $\mathrm{E}$ \\
\hline Dryopteridaceae & Elaphoglossum minutum (Pohl ex Fée) T. Moore & MF & $\mathrm{E}$ \\
\hline Dryopteridaceae & Lastreopsis amplissima (C. Presl) Tindale & $\mathrm{LF}, \mathrm{MF}$ & $\mathrm{T}$ \\
\hline Dryopteridaceae & Mickelia scandens (Raddi) R.C. Moran et al. & $\mathrm{LF}, \mathrm{SF}, \mathrm{MF}$ & $\mathrm{E}, \mathrm{H}, \mathrm{R}$ \\
\hline Dryopteridaceae & Olfersia cervina (L.) Kunze & LF, SF & $\mathrm{T}, \mathrm{R}$ \\
\hline Dryopteridaceae & EA Polybotrya semipinnata Fée & SF & $\mathrm{H}$ \\
\hline Dryopteridaceae & EA Polybotrya cylindrica Kaulf. & $\mathrm{RF}, \mathrm{LF}, \mathrm{SF}, \mathrm{MF}$ & $\mathrm{H}$ \\
\hline Dryopteridaceae & 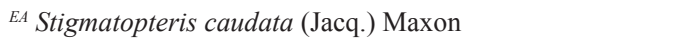 & SF & $\mathrm{T}$ \\
\hline Dryopteridaceae & ${ }^{E A}$ Stigmatopteris heterocarpa (Fée) Rosenst. & LF, SF & $\mathrm{T}$ \\
\hline Gleicheniaceae & Gleichenella pectinata (Willd.) Ching & $\mathrm{LF}$ & $\mathrm{T}$ \\
\hline Gleicheniaceae & Sticherus bifidus (Willd.) Ching & MF & $\mathrm{T}$ \\
\hline Gleicheniaceae & ${ }^{E B}$ Sticherus nigropaleaceus (Sturm) J. Prado \& Lellinger & $\mathrm{LF}, \mathrm{SF}$ & $\mathrm{T}$ \\
\hline Hemidictyaceae & Hemidictyum marginatum (L.) C. Presl & $\mathrm{LF}$ & $\mathrm{T}$ \\
\hline Hymenophyllaceae & Abrodictyum rigidum (Sw.) Ebihara \& Dubuisson & $\mathrm{LF}, \mathrm{SF}, \mathrm{MF}$ & $\mathrm{T}, \mathrm{R}$ \\
\hline Hymenophyllaceae & Didymoglossum hymenoides (Hedw.) Desv. & $\mathrm{RF}$ & $\mathrm{E}$ \\
\hline Hymenophyllaceae & Didymoglossum krausii (Hook. \& Grev.) C. Presl & $\mathrm{LF}, \mathrm{MF}$ & $\mathrm{E}$ \\
\hline Hymenophyllaceae & Didymoglossum reptans (Sw.) C. Presl & LF, SF & $\mathrm{E}$ \\
\hline Hymenophyllaceae & Hymenophyllum asplenioides (Sw.) Sw. & MF & $\mathrm{E}$ \\
\hline Hymenophyllaceae & Hymenophyllum caudiculatum Mart. & MF & $\mathrm{E}$ \\
\hline Hymenophyllaceae & Hymenophyllum hirsutum (L.) Sw. & $\mathrm{RF}, \mathrm{MF}$ & $\mathrm{E}$ \\
\hline Hymenophyllaceae & Hymenophyllum polyanthos (Sw.) Sw. & $\mathrm{RF}, \mathrm{SF}, \mathrm{MF}$ & $\mathrm{E}$ \\
\hline Hymenophyllaceae & Hymenophyllum rufum Fée & MF & $\mathrm{E}$ \\
\hline Hymenophyllaceae & Polyphlebium angustatum (Carmich.) Ebihara \& Dubuisson & MF & $\mathrm{E}$ \\
\hline Hymenophyllaceae & Polyphlebium pyxidiferum (L.) Ebihara \& Dubuisson & MF & $\mathrm{E}$ \\
\hline Hymenophyllaceae & Trichomanes cristatum Kaulf. & $\mathrm{RF}$ & $\mathrm{E}$ \\
\hline Hymenophyllaceae & Trichomanes polypodioides L. & MF & $\mathrm{E}$ \\
\hline Hymenophyllaceae & Vandenboschia radicans (Sw.) Copel. & $\mathrm{LF}, \mathrm{SF}, \mathrm{MF}$ & $\mathrm{E}$ \\
\hline Lindsaeaceae & Lindsaea divaricata Klotzsch & $\mathrm{SF}, \mathrm{MF}$ & $\mathrm{T}$ \\
\hline Lindsaeaceae & Lindsaea lancea var.lancea (L.) Bedd. & $\mathrm{RF}, \mathrm{LF}, \mathrm{MF}$ & $\mathrm{T}$ \\
\hline Lindsaeaceae & ${ }^{E B}$ Lindsaea quadrangularis Raddi subsp. quadrangularis & $\mathrm{RF}, \mathrm{SF}, \mathrm{MF}$ & $\mathrm{T}$ \\
\hline Lindsaeaceae & ${ }^{E A}$ Lindsaea virescens $\mathrm{Sw}$. var. virescens & MF & $\mathrm{T}$ \\
\hline Lomariopsidaceae & ${ }^{E A}$ Lomariopsis marginata (Schrad.) Kuhn & LF, SF & $\mathrm{H}$ \\
\hline Lomariopsidaceae & ${ }^{E B} N e p h r o l e p i s$ biserrata (Sw.) Schott & $\mathrm{RF}, \mathrm{LF}$ & $\mathrm{E}$ \\
\hline Lomariopsidaceae & Nephrolepis cordifolia (L.) C. Presl & LF & $\mathrm{E}$ \\
\hline Lomariopsidaceae & Nephrolepis rivularis (Vahl) Mett. ex Krug & LF, SF & $\mathrm{E}$ \\
\hline Lygodiaceae & Lygodium volubile Sw. & $\mathrm{LF}, \mathrm{SF}$ & $\mathrm{S}$ \\
\hline Marattiaceae & 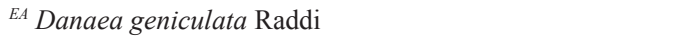 & $\mathrm{LF}, \mathrm{SF}, \mathrm{MF}$ & $\mathrm{T}$ \\
\hline Polypodiaceae & Campyloneurum angustifolium (Sw.) Fée & MF & $\mathrm{E}$ \\
\hline Polypodiaceae & ${ }^{E A}$ Campyloneurum decurrens C. Presl & LF, SF & $\mathrm{E}, \mathrm{R}$ \\
\hline Polypodiaceae & ${ }^{E A}$ Campyloneurum lapathifolium (Poir.) Ching & MF & $\mathrm{E}$ \\
\hline Polypodiaceae & Campyloneurum minus Fée & LF, SF & $\mathrm{E}$ \\
\hline Polypodiaceae & Campyloneurum nitidum (Kaulf.) C. Presl & $\mathrm{RF}, \mathrm{LF}, \mathrm{SF}, \mathrm{MF}$ & $\mathrm{E}, \mathrm{R}$ \\
\hline Polypodiaceae & Campyloneurum rigidum J. Sm. & $\mathrm{RF}, \mathrm{MF}$ & $\mathrm{E}$ \\
\hline Polypodiaceae & ${ }^{E A}$ Cochlidium punctatum (Raddi) L.E. Bishop & MF & $\mathrm{E}$ \\
\hline Polypodiaceae & Cochlidium serrulatum (Sw.) L.E. Bishop & $\mathrm{RF}, \mathrm{MF}$ & $\mathrm{E}$ \\
\hline
\end{tabular}


Table 1. Continued...

\begin{tabular}{|c|c|c|c|}
\hline Family & Species & Forest type & Life form \\
\hline Polypodiaceae & Melpomene pilosissima (M. Martens \& Galeotti) A.R. Sm. \& R.C. Moran & MF & $\mathrm{E}$ \\
\hline Polypodiaceae & Microgramma geminata (Schrad.) R.M. Tryon \& A.F. Tryon & RF, LF & $\mathrm{E}$ \\
\hline Polypodiaceae & Microgramma percussa (Cav.) de la Sota & SF & $\mathrm{E}$ \\
\hline Polypodiaceae & ${ }^{E B}$ Microgramma tecta (Kaulf.) Alston var. tecta & $\mathrm{LF}, \mathrm{SF}, \mathrm{MF}$ & $\mathrm{E}$ \\
\hline Polypodiaceae & Microgramma vacciniifolia (Langsd. \& Fisch.) Copel. & $\mathrm{RF}$ & $\mathrm{E}$ \\
\hline Polypodiaceae & 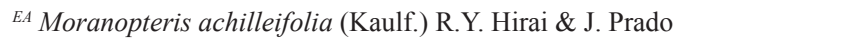 & $\mathrm{MF}$ & $\mathrm{E}$ \\
\hline Polypodiaceae & Niphidium crassifolium (L.) Lellinger & MF & $\mathrm{E}$ \\
\hline Polypodiaceae & Pecluma chnoophora (Kunze) Salino \& Costa Assis & LF & $\mathrm{E}$ \\
\hline Polypodiaceae & 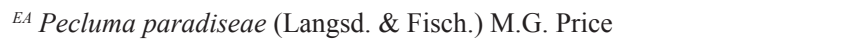 & MF & $\mathrm{E}$ \\
\hline Polypodiaceae & ${ }^{E A}$ Pecluma recurvata (Kaulf.) M.G. Price & $\mathrm{MF}$ & $\mathrm{E}$ \\
\hline Polypodiaceae & 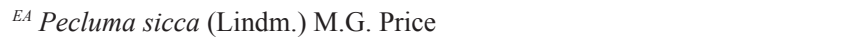 & $\mathrm{MF}$ & $\mathrm{E}$ \\
\hline Polypodiaceae & Pleopeltis astrolepis (Liebm.) E. Fourn. & $\mathrm{MF}$ & $\mathrm{E}$ \\
\hline Polypodiaceae & Pleopeltis furcata (L.) A.R.Sm. & SF & $\mathrm{E}$ \\
\hline Polypodiaceae & Pleopeltis hirsutissima (Raddi) de la Sota & LF & $\mathrm{E}$ \\
\hline Polypodiaceae & 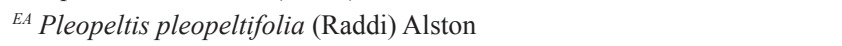 & SF & $\mathrm{E}$ \\
\hline Polypodiaceae & Serpocaulon catharinae (Langsd. \& Fisch.) A.R. Sm. & $\mathrm{MF}$ & $\mathrm{E}$ \\
\hline Polypodiaceae & Serpocaulon fraxinifolium (Jacq.) A.R. Sm. & $\mathrm{LF}, \mathrm{SF}, \mathrm{MF}$ & $\mathrm{E}$ \\
\hline Polypodiaceae & 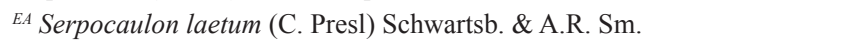 & $\mathrm{MF}$ & $\mathrm{E}$ \\
\hline Polypodiaceae & 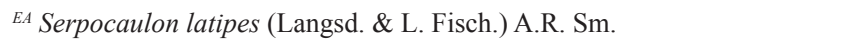 & $\mathrm{LF}, \mathrm{MF}$ & $\mathrm{E}$ \\
\hline Polypodiaceae & ${ }^{E A}$ Serpocaulon menisciifolium (Langsd. \& Fisch.) A.R. Sm. & MF & $\mathrm{E}$ \\
\hline Polypodiaceae & ${ }^{E A}$ Terpsichore chrysleri (Proctor ex Copel.) A.R. Sm. & MF & $\mathrm{E}$ \\
\hline Pteridaceae & Acrostichum danaeifolium Langsd. \& Fisch. & LF & $\mathrm{T}$ \\
\hline Pteridaceae & Adiantum windischii J. Prado & LF & $\mathrm{T}$ \\
\hline Pteridaceae & Anetium citrifolium (L.) Splitg. & SF & $\mathrm{E}$ \\
\hline Pteridaceae & Doryopteris concolor (Langsd. \& Fisch.) Kuhn & LF & $\mathrm{T}$ \\
\hline Pteridaceae & Pityrogramma calomelanos (L.) Link & LF & $\mathrm{T}$ \\
\hline Pteridaceae & Pteris decurrens C. Presl & $\mathrm{LF}, \mathrm{SF}, \mathrm{MF}$ & $\mathrm{T}$ \\
\hline Pteridaceae & Pteris splendens Kaulf. & $\mathrm{MF}$ & $\mathrm{T}$ \\
\hline Pteridaceae & Pteris deflexa Link & $\mathrm{MF}$ & $\mathrm{T}$ \\
\hline Pteridaceae & Radiovittaria stipitata (Kunze) E.H.Crane & $\mathrm{RF}, \mathrm{MF}$ & $\mathrm{E}$ \\
\hline Saccolomataceae & Saccoloma brasiliense (C. Presl) Mett. & LF, SF & $\mathrm{T}$ \\
\hline Selaginellaceae & Selaginella flexuosa Spring & LF & $\mathrm{R}, \mathrm{T}$ \\
\hline Selaginellaceae & ${ }^{E A}$ Selaginella macrostachya (Spring) Spring & $\mathrm{MF}$ & $\mathrm{T}, \mathrm{R}$ \\
\hline Selaginellaceae & Selaginella muscosa Spring & LF & $\mathrm{T}$ \\
\hline Selaginellaceae & Selaginella sulcata (Desv. ex Poir.) Spring ex Mart. & LF, SF & $\mathrm{T}$ \\
\hline Tectariaceae & Tectaria incisa Cav. & LF, SF & $\mathrm{T}$ \\
\hline Thelypteridaceae & Macrothelypteris torresiana (Gaudich.) Ching & LF & $\mathrm{T}$ \\
\hline Thelypteridaceae & Thelypteris angustifolia (Willd.) Proctor & LF & $\mathrm{T}$ \\
\hline Thelypteridaceae & EA Thelypteris eriosora (Fée) Ponce & $\mathrm{MF}$ & $\mathrm{T}$ \\
\hline Thelypteridaceae & Thelypteris interrupta (Willd.) K. Iwats. & LF & $\mathrm{T}$ \\
\hline Thelypteridaceae & Thelypteris longifolia (Desv.) R.M. Tryon & $\mathrm{RF}$ & $\mathrm{T}$ \\
\hline Thelypteridaceae & ${ }^{E A}$ Thelypteris metteniana Ching & LF & $\mathrm{T}$ \\
\hline Thelypteridaceae & Thelypteris opposita (Vahl) Ching & LF & $\mathrm{T}$ \\
\hline Thelypteridaceae & Thelypteris salzmannii (Fée) C.V. Morton & LF & $\mathrm{T}$ \\
\hline
\end{tabular}

Among the guilds of life forms, the epiphytes were predominant, with 62 species overall (Figure 2). According to Madison (1977), the high diversity of epiphytes in neotropical forests is favored by an abundance of favorable habitats, especially in montane regions. In these regions, these groups of plants represent about $50 \%$ of the total flora (Kelly et al., 1994), due, primordially, to the forest structure and water availability (Grubb \& Withimore, 1966).

The high degree of endemism for south and southeastern Brazil (Tryon \& Tryon, 1982; Prado \& Silvestre, 2010) can be exemplified in this work, which presents about $30.37 \%$ (41 spp.) of the taxa considered endemic from Brazil. About $85 \%$ (35 spp.) of these are endemic to the Atlantic Rain Forest (Figure 3).
Two invasive exotic species, Macrothelypteris torresiana (Gaudich.) Ching and Nephrolepis cordifolia (L.) C. Presl, were found in the study area. These species are common in various other sites of the Atlantic Forest (Zenni \& Ziller, 2011).

In total, five species are considered endangered in the vulnerable category, according to "Lista oficial de espécies ameaçadas de extinção no Estado de São Paulo" (Resolução SMA no 48/2004): Anetium citrifolium (L.) Splitg., Asplenium muellerianum Rosenst., Cyathea glaziovii (Fée) Domin, Elaphoglossum macahense (Fée) Rosenst., and Thelypteris angustifolia (Willd.) Proctor. This factor stresses the importance of the Picinguaba and Santa Virgínia Nuclei for the conservation of biodiversity in the State of São Paulo. 


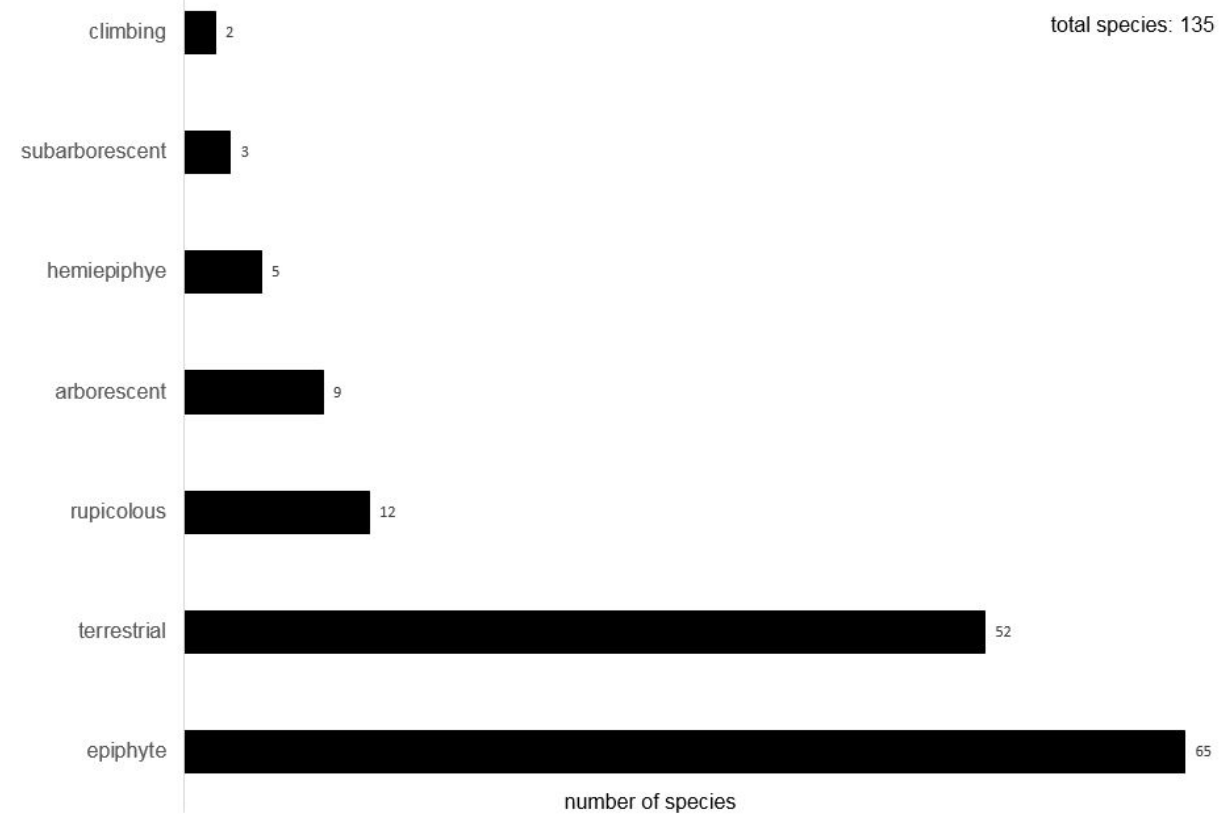

Figure 2. Species number per different life forms in the Picinguaba and Santa Virginia Nuclei.

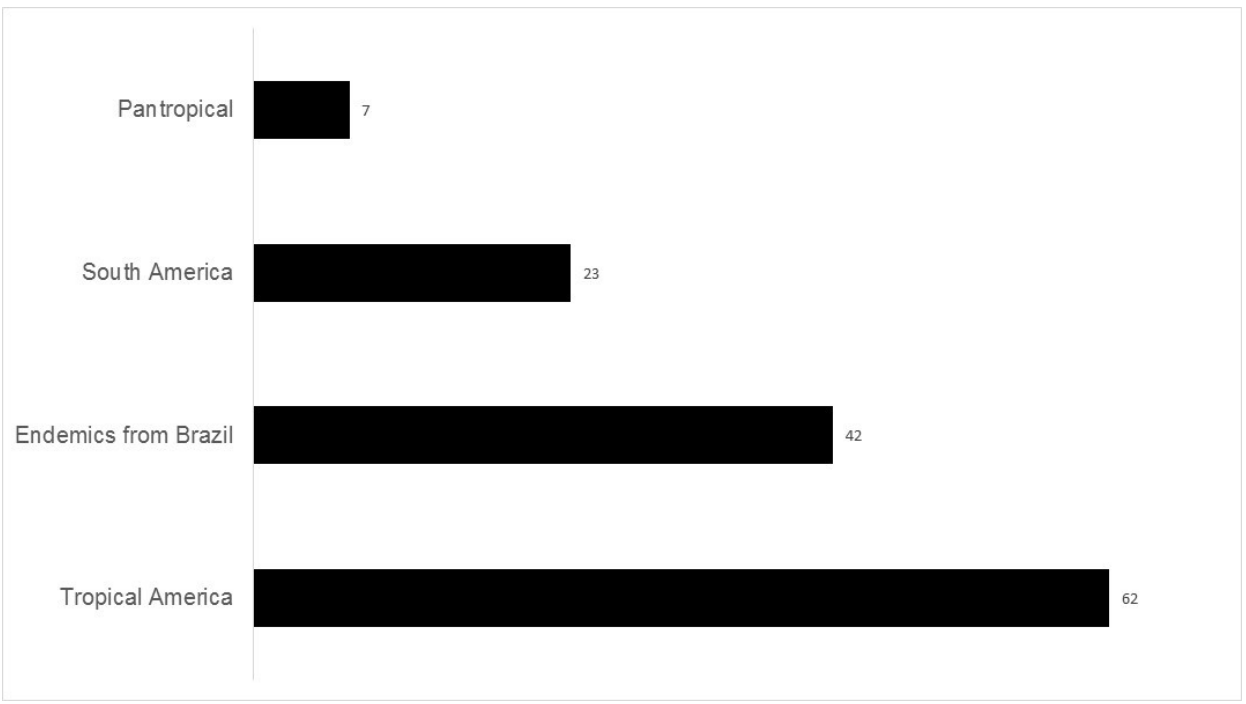

Figure 3. Species number found in PESM area, separated by geographic distribution class.

This forest is lush and diverse, with humidity and relief suitable for the development of ferns and lycophytes. Thus, the present paper contributes to the knowledge of these groups and highlights the importance of the permanent preservation of these areas.

The key presented ahead is the first one for a broad area covered by the Atlantic Rain Forest in Brazil. It constitutes an important tool for the identification of ferns and lycophytes from the northeastern portion of the state of São Paulo.

\section{Acknowledgments}

The first author is grateful to the CNPq for the scholarship and Biota FAPESP Program for financial support. We also thank Fernando B. Matos for English corrections to improve the manuscript.

\section{References}

ASSIS M.A. 1999. Florística e caracterização das comunidades vegetais da Planície Costeira de Picinguaba, Ubatuba/SP. Tese de Doutorado, Universidade Estadual de Campinas, Campinas.

ATHAYDE-FILHO F.P., PEREIRA. V.S., SMIDT E.C. \& NONATO F.R. 2003 Pteridófitas do Parque Estadual da Ilha Anchieta (PEIA). Bradea 12: 55-66.

BOLDRIN A.H.L. \& PRADO J. 2007. Pteridófitas terrestres e rupícolas do Forte dos Andradas, Guarujá, São Paulo, Brasil. Boletim de Botânica da Universidade de São Paulo 25: 1-69.

CHRISTENHUSZ J. M. M., ZHANG XIAN-CHUN \& SCHNEIDER H. 2011 A linear sequence of extant families and genera of lycophytes and ferns. Phytotaxa 19: 7-54.

CUSTÓDIO FILHO A. 1989. Flora da Estação Ecológica de Boracéia - Listagem de espécies. Revista do Instituto Florestal 1: 161-199. 
FORZZA R.C., BAUMGRATZ J.F.A., BICUDO C.E.M., CANHOS D.A.I., CARVALHO JR. A.A., COELHO M.A.N., COSTAA.F., COSTA D.P., HOPKINS M.G., LEITMAN P.M., LOHMANN L.G., LUGHADHA E.N., MAIA L.C., MARTINELLI G., MENEZES M., MORIM M.P., PEIXOTO A.L., PIRANI J.R., PRADO J., QUEIROZ L.P., SOUZA S., SOUZA V.C., STEHMANN J.R., SYLVESTRE L.S., WALTER B.M.T. \& ZAPPI D.C. 2012. New Brazilian Floristic List Highlights Conservation Challenges. BioScience 62(1): 39-45.

FUNDAÇÃO SOS MATA ATLÂNTICA \& INSTITUTO NACIONAL DE PESQUISAS ESPACIAIS - INPE. 2011. Atlas dos Remanescentes Florestais da Mata Atlântica. <http://mapas.sosma.org.br/>. Acesso em 30 de julho de 2011.

GRUBB, P.J. \& WITHIMORE, T.C. 1966. A comparison of montane and Lowland Rain forest in Ecuador, II. The climate and its Effects on the Distribution and Physiognomy of the Forest. J Ecol 54: 303-333.

HOLTTUM R.E. 1938. The ecology of tropical pteridophytes. In: Manual of Pteridology (F. Verdoorn \& A.H.G. Alston, eds.) Springer Netherlands, Plant Sciences, p. 420-450.

JOLY C.A., AIDAR. M.P.M., KLINK C.A., MCGRATH D.G., MOREIRA A.G., MOUTINHO P., NEPSTAD D.C., OLIVEIRAA.A., POTT A., RODAL M.J.N. \& SAMPAIO E.V.S.B. 1999. Evolution of the Brazilian phytogeography classification systems: implications for biodiversity conservation. Ciência e cultura 51 (5-6): 331-348.

KELLY, D.L.; TANNER, E.V.J.; LUGHADHA NIC, E.M. \& KAPOS, V. 1994. Floristic and biogeography of a rain forest in the Venezuelan Andes. J Biogeogr 21: 421-440.

LISTA DE ESPÉCIES DAFLORA DO BRASIL. Jardim Botânico do Rio de Janeiro. Disponível em: <http://floradobrasil.jbrj.gov.br/>. Acesso em: 13 Abr. 2015.

MADISON, M. 1977. Vascular epiphytes: Their systematic occurrence and salient features. Selbyana 2: 1-13.

MAZZIERO.F.F.F.; LABIAK, P.H. \& PACIENCIA, M.L.B. 2015. Checklist of ferns and lycophytes from the Parque Estadual Turístico do Alto Ribeira, Iporanga, São Paulo, Brazil. Check List 11: 1791.

MORAN R.C. 2008. Biogeography of ferns and lycophytes. In: The Biology and Evolution of Ferns and Lycophytes (C. Haufler \& T.A Ranker, eds.) Cambridge University Press, p. 369-396.

MYERS N., MITTERMEIER R.A., MITTERMEIER C.G., FONSECA G.A.B. \& KENT J. 2000. Biodiversity hotspots for conservation priorities. Nature $403: 853-858$

PACIENCIA, M.L.B. 2008. Diversidade de pteridófitas em gradientes de altitude na Mata Atlântica do Estado do Paraná, Brasil. Tese de Doutorado. Universidade de São Paulo, São Paulo, 229p.

PAGE C.N. 1979. The diversity of ferns: an ecological perspective. In: Dyer A.F. (ed.). The experimental biology of ferns. Academic Press, London, UK, p. 9-56.

PAGE C.N. 1979. The diversity of ferns: an ecological perspective. In: Dyer A.F. (ed.). The experimental biology of ferns. Academic Press, London, UK, p. 9-56.

PRADO J. \& LABIAK P.H. 2001. Pteridófitas. In: M. C. H. Mamede, I. Cordeiro \& L. Rossi, Flora vascular da Serra da Juréia, Município de Iguape, São Paulo, Brasil. Boletim do Instituto de Botânica 15: 83-86.

PRADO J. \& LABIAK P.H. 2009. Pteridófitas da Reserva Biológica de Paranapiacaba. In: M.I.M.S. Lopes; M. Kirizawa; M.M.R.F.de Melo. (Org.). Patrimônio da Reserva Biológica do Alto da Serra de Paranapiacaba: A Antiga Estação Biológica do Alto da Serra. São Paulo: Editora Secretaria do Meio Ambiente de São Paulo, p. 269-290.
PRADO J. 2004. Pteridófitas do Maciço da Juréia. In: W. Duleba \& O.A.V. Marques (orgs.) Estação Ecológica Juréia - Itatins: ambiente físico, flora e fauna. 1a. ed. FAPESP, Instituto Butantan, Holos, Ribeirão Preto, p. 139-151.

PRADO, J, SYLVESTRE L. S., LABIAK P. H., WINDISCH P. G., SALINO A., BARROS I. C. L., HIRAI R. Y., ALMEIDA T. E., SANTIAGO A. C. P., KIELING-RUBIO M. A., PEREIRAA. F. N., ØLLGAARD B., RAMOS C. G. V., MICKEL J. T., DITTRICH V. A. O., MYNSSEN C. M., SCHWARTSBURD P. B., CONDACK J. P. S., PEREIRA J. B. S., MATOS F. B. 2015. Diversity of ferns and lycophytes in Brazil. Rodriguésia 66(4): 1073-1083.

PRADO, J. \& SYLVESTRE, L.S. 2010. As samambaias e licófitas do Brasil. In: Forzza, R.C.; Baumgratz, J.F.A.; Bicudo, C.E.M.; Canhos, D.A.I.; Carvalho, Jr. A.A.; Coelho, M.A.N.; Costa, A.F.; Costa, D.P.; Hopkins, M.G.; Leitman, P.M.; Lohmann, L.G.; Lughadha, E.N.; Maia, L.C.; Martinelli, G.; Menezes, M.; Morim, M.P.; Peixoto, A.L.; Pirani, J.R.; Prado, J.; Queiroz, L.P.; Souza, S.; Souza, V.C.; Stehmann, J.R.; Sylvestre, L.S.; Walter, B.M.T. \& Zappi, D.C. (Org.). Catálogo de plantas e fungos do Brasil. Rio de Janeiro: Andrea Jakobsson Estúdio; Jardim Botânico do Rio de Janeiro. Pp. 69-74.

PRADO, J. 1998. Pteridófitas do Estado de São Paulo. In: Bicudo, C.E.M. \& Shepherd G. (Eds.). Fungos macroscópicos e plantas do Estado de São Paulo (Série Biodiversidade do Estado de São Paulo). São Paulo: FAPESP 2: 49-61.

ROOS M. 1996. Mapping the world's pteridophyte diversity - systematics and floras. In: Pteridology in Perspective, ed. J. M. Camus, M. Gibby and R. J. Johns. Kew, UK: Royal Botanic Gardens, p. 29-42.

SALEMI L.F. 2009. Balanço de água e de nitrogênio em uma microbacia coberta de pastagens no litoral norte do Estado de São Paulo. 2009. Dissertação de Mestrado, Universidade de São Paulo, Piracicaba.

SALINO, A. \& ALMEIDA, T.E. 2008. Pteridófitas do Parque Estadual do Jacupiranga, SP, Brasil. Acta Botanica Brasilica 22: 983-991.

SETZER, J. 1966. Atlas climatológico do estado de São Paulo. Comissão Interestadual da Bacia do Paraná-Paraguai; CESP, São Paulo.

SILVAA.T. 1989. Pteridófitas. In: O. Fidalgo \& V.L.R. Bononi (coords.). Técnicas de coleta, preservação e herborização de material botânico. Manual no 04.2 ed. São Paulo, Instituto de Botânica, p. 32 -34.

SMITH A.R., PRYER K.M., SCHUETTPELZ E. KORALL P., SCHNEIDER, H. \& WOLF P.G. 2006. A classification for extant ferns. Taxon 55: 705-731.

SMITH A.R., PRYER K.M., SCHUETTPELZ E., KORALL P., SCHNEIDER H. \& WOLF P.G. 2008. Fern Classification. In: T.A. Ranker \& C.H. Haufler (eds.). Biology and evolution of ferns and Lycophytes. Cambridge University, Cambridge, p. 417-467.

TRYON, R. M. 1986. Some new names and new combinations in Pteridaceae. American Fern Journal 76(4): 184-186.

TRYON, R.M. \& TRYON, A.F. 1982. Ferns and allied plants, with special reference to tropical America. Springer-Verlag, New York. 857p.

VELOSO H.P., RANGEL FILHO A.L.R. \& LIMA J.C.A. 1991. Classificação da Vegetação Brasileira, Adaptada a um Sistema Universal. IBGE, Departamento de Recursos Naturais e Estudos Ambientais.

ZENNI R.D. \& ZILLER S.R. 2011. An overview of invasive plants in Brazil. Revista Brasileira de. Botânica 34(3): 431-446. 
Nóbrega, G.A. et al.

\section{Identification key for lycophytes and ferns species found in Biota Gradient Functional areas (Picinguaba and Santa Virgínia Nuclei)}

1. Arborescent plants (tree ferns)

2. Petiole base with black, tapered and rigid spines; thickly coated with filamentous setae scales

3. Aphlebias present; petiole base persistent on the stem Alsophila setosa

3. Aphlebias absent; petiole base deciduous leaving a scar on the stem Alsophila sternbergii

2. Petiole base with spines with the same coloration of this, conical, acute apex or absents; lanceolate scale scattered or concentrated in proximal portion, setae present or absent

4. Petiole scales whitish or bicolor (i.e., whitish at margins and brown at center)

5. Petiole base deciduous leaving a scar on the stem, scales with black setae

Cyathea hirsuta

5. Petiole base persistent on the stem, scales without setae

6. Petiole scales concolor (whitish)

Cyathea leucofolis

6. Petiole scales bicolor (whitish and brown)

Cyathea dichromatolepis

4. Petiole scales brown

7. Petiole base deciduous leaving a scar on the stem, indusium globose

Cyathea delgadii

7. Petiole base persistent on the stem, indusium absent

8. Secondary veins predominantly forked; costae and costules only with inflated scales

Cyathea phalerata

8. Secondary veins predominantly simple; costae and costules with plan and inflated scales (bullate scales)

9. Petiole with spines at base; sori with paraphyses longer than the sporangia Cyathea atrovirens

9. Petiole without spines (with small round projections); sori with paraphyses shorter than the sporangia Cyathea glaziovii

1. Herbaceous plants (climbers, epiphytes, hemiepiphytes, terrestrial, rupicolous, or subarborescent)

10. Leaves with a single vein (microphylls) and with a minute ligule; stems with rhizophores

11. Stems articulate; lateral and axillary microphylls with base auriculate.... Selaginella sulcata

11. Stems not articulate, lateral and axillary microphylls not auriculate at base

12. Microphylls with ciliate margins Selaginella macrostachya

12. Microphylls without ciliate margins

13. Lateral microphylls oval; dorsal microphylls asymmetric Selaginella muscosa

13. Lateral microphylls oblong or oblong-elliptic; dorsal microphylls symmetric Selaginella flexuosa

10. Leaves with more than one vein (megaphylls), without ligule; rhizome or stem with adventitious roots, without rhizophores

14. Lamina undivided (or only fertile leave undivided in Cochlidium serrulatum), without segments (not pinnate)

15. Sori round, oblong or ovate; venation anastomosing with free veins included in the areoles

16. Stem scales not clathrate

17. Lamina without scales Microgramma geminata

17. Lamina with scales

18. Scales present only on the adaxial surface

Microgramma vacciniifolia

18. Scales present on both surfaces

19. Sterile leaf linear-lanceolate Microgramma percussa

19. Sterile leaf ovate

Microgramma tecta

16. Stem scales clathrate

20. Sori oblong or ovate, longer than wide Pleopeltis astrolepis 
20. Sori round

21. One row of sori between two lateral veins

Niphidium crassifolium

21. Two rows of sori between two lateral veins

22. Lateral veins abaxially prominent

23. Stem scales brown, ovate, sub-globose

Campyloneurum nitidum

23. Stem scales slightly bicolor (brown and light-brown), lanceolate

Campyloneurum minus

22. Lateral veins abaxially not prominent

24. Rhizome with peltate scales; lamina margins revolute

Campyloneurum angustifolium

24. Rhizome with scales basifix, cordate; lamina margins flat

25. Lamina linear, rigid and bright.

Campyloneurum rigidum

25. Lamina lanceolate, flexible, opaque

Campyloneurum lapathifolium

15. Sori not round, oblong or oval; veins free or if anastomosing without free veins included in the areoles

26. Sori acrostichoid

27. Lamina elliptic, oblong or ovate

28. Apex of the lamina round .

Elaphoglossum lingua

28. Apex of the lamina acuminate or acute

29. Stem scales yellow or light brown, ovate

Elaphoglossum minutum

29. Stem scales black, fimbriate, with irregular process and cilia

Elaphoglossum luridum

27. Lamina lanceolate

30. Lamina without scales on laminar tissue (present only on the petiole, costae, and margins)

Elaphoglossum decoratum

30. Lamina with scales on laminar tissue (including petiole and costae) or lamina glabrous

31. Lamina glabrous or with scales, the scales not similar to those of the petiole

32. Veins free near the margins of the lamina

Elaphoglossum macahense

32. Veins anastomosing near the margins of the lamina

Elaphoglossum macrophyllum

31. Lamina with scales, the scales similar to those of the petiole

33. Scales of the lamina linear-lanceolate

Elaphoglossum horridulum

33. Scales of the lamina oblong-lanceolate or lanceolate

Elaphoglossum chrysolepis

26. Sori not acrostichoid (sporangia forming lines parallel to the veins, costae, and margins or immersed, or on the surface forming coenosorus)

34. Venation free

35. Lamina shorter than $5 \mathrm{~cm}$ long; sporangia forming coenosorus; spores with chlorophyll

36. Hydathodes inconspicuous on the adaxial surface of the lamina; sori restricted to the distal pat of the lamina

Cochlidium serrulatum

36. Hydathodes conspicuous on the adaxial surface of the lamina; sori not restricted to the distal pat of the lamina

Cochlidium punctatum

35. Lamina bigger than $5 \mathrm{~cm}$ long; sporangia linear; spores without chlorophyll

37. Lamina $>4 \mathrm{~cm}$ wide; sori parallel to the lateral veins Asplenium serratum

37. Lamina $<3 \mathrm{~cm}$ wide; sori parallel to the costa

Blechnum lanceola

34. Venation anastomosing, without free veins included in the areoles

38. Lamina with only one row of areoles between the margin and costa

Radiovittaria stipitata

38. Lamina with several rows of areoles between the margin and costa

Anetium citrifolium

14. Lamina divided (pinnate, 2-pinnate or more divided, pinnatifid, pinnatisect, or dichotomous) 
39. Lamina with 1 or 2 cell layers in thickness, translucid

40. Indusium bivalve, receptacle not extended beyond the margins of the involucre

41. Lamina glabrous

42. Lamina 1-pinnate-pinnatisect, petiole not winged

Hymenophyllum asplenioides

42. Lamina 2 or 3-pinnate-pinnatissect, petiole winged

43. Terminal segments long (caudate), plants $>10 \mathrm{~cm}$ long Hymenophyllum caudiculatum

43. Terminal segments shorts (not caudate), plants $<10 \mathrm{~cm}$ long

Hymenophyllum polyanthos

41. Lamina with hairs

44. Rachis fully winged; fronds with stellate hairs on the petiole, rachis, veins, and margins of the lamina (except on the laminar tissue between veins and margins) Hymenophyllum hirsutum

44. Rachis irregular winged; fronds with stellate hairs on the entire blade (including the laminar tissue).....

Hymenophyllum rufum

40. Indusium tubular and bilabiate, receptacle long beyond involucre margins

45. Plants terrestrial; rhizome erect or short-creeping; fronds fasciculate

46. Lamina deltoid and 3 or 4-pinate-pinnatisect, rachis without wings Abrodictyum rigidum

46. Lamina lanceolate and 1-pinate-pinatissect, rachis with narrow wings Trichomanes cristatum

45. Plants epiphytes; rhizome long-creeping; fronds not fasciculate

47. False veins present in the laminar tissue

48. Indusium included in the lamina; false veins parallel to the margin of the lamina

Didymoglossum krausii

48. Indusium exserted; false veins not parallel to the margin of the lamina

49. Plants up to $5 \mathrm{~cm}$ long; margins of the lamina with furcate and stellate hairs, restricted to sinus

Didymoglossum reptans

49. Plants up to $2 \mathrm{~cm}$ long; margins of the lamina with only furcate hairs Didymoglossum hymenoides

47. False veins absent in the laminar tissue

50. Venation catadromous

Trichomanes polypodioides

50. Venation anadromous

51. Petiole not winged Polyphlebium angustatum

51. Petiole winged

52. Stem and petiole with brown hairs Vandenboschia radicans

52. Stem and petiole with black hairs

Polyphlebium pyxidiferum

39. Lamina with more than 2 cell layers in thickness, not translucid

53. Petiole base persistent in the stem, forming phyllopodium

54. Lamina dichotomously divided

55. Sori rounded or oblong Pleopeltis pleopeltifolia

55. Sori linear Pleopeltis furcata

54. Lamina pinnate, 2-pinnate or more divided

56. Petiole cylindrical, not sulcate

57. Veins sim Pecluma sicca

\section{Veins forked}

58. Proximal segments reduced (auriculate) Pecluma paradiseae

58. Proximal segments not reduced Pecluma recurvata

56. Petiole sulcate 
59. Venation free

60. Scales abundant on the laminar tissue Pleopeltis hirsutissima

60. Scales absent on the laminar tissue Pecluma chnoophora

59. Venation anastomosing

61. Lamina pinnatisect

62. Stem dark-brown to black, pruinose Serpocaulon catharinae

62. Stem light brown to yellow, without waxy deposits

63. Lamina without hairs, scales presents in the costae Serpocaulon latipes 63. Lamina with hyaline hairs Serpocaulon laetum

\section{Lamina 1-pinnate}

64. Medial pinnae not adnate Serpocaulon fraxinifolium

64. Medial pinnae with the acroscopic side slightly adnate Serpocaulon meniscifolium

53. Phyllopodium absent

65. Sori linear

66. Sori marginal or submarginal

67. Sori submarginal, protected by an abaxial indusium

68. Pinnules 2 or 3 times forked Lindsaea virescens var. virescens

68. Pinnules not forked (pinnate or more divided)

69. Rachis and secondary rachis quadrangular Lindsaea quadrangularis subsp. quadrangularis

69. Rachis and secondary rachis not quadrangular

70. Rachis and secondary rachis paleaceous, winged paleaceous; pinnules semilunate

Lindsaea lancea var. lancea

70. Rachis and secondary rachis reddish brown, winged paleaceous contracting with rachis color; pinnules not semilunate Lindsaea divaricata

67. Sori marginal, protected by an adaxial indusium formed by the revolute margin of the lamina

71. Lamina not fully pinnate

Doryopteris concolor

71. Lamina pinnate

72. Lamina 1-pinnate Pteris splendens

72. Lamina 2-3-pinnate

73. Venation free Pteris deflexa

73. Venation anastomosing Pteris decurrens

66. Sori not marginal and not submarginal

74. Sori parallel and adjacent to the costa

75. Plants with indeterminate growth, climbing Salpichlaena volubilis

75. Plants with determinate growth, not climbing

76. Fertile and sterile fronds monomorphic

77. Proximal pinnae gradually reduced toward the lamina base

78. Plants herbaceous; stem stoloniferous; petiole with lanceolate and brownish scales Blechnum polypodioides

78. Plants sub-arborescent; stem not stoloniferous; petiole with linear to linear-lanceolate and blackish scales Blechnum brasiliense

77. Proximal pinnae not reduced or only slightly reduced toward the lamina base 
79. Fronds entire

Blechnum lanceola

79. Fronds pinnate

80. Proximal pinnae petiolulate Blechnum gracile

80. Proximal pinnae sessile or adnate Blechnum occidentale

76. Fertile and sterile fronds dimorphic

81. Petiole base with concolor scales Blechnum cordatum

81. Petiole base with bicolor scales

82. Stem erect, subarborescent with scales, the scales blackish or brown in the center and golden near the margin Blechnum schomburgkii

82. Stem scandent with scales, the scales blackish or brown in the center and light brown near the margin Blechnum acutum

74. Sori not parallel to the costa

83. Sori in both sides of the secondary vein, at least in the proximal veins

84. Lateral pinnae entire Diplazium riedelianum

84. Lateral pinnae divided Diplazium cristatum

83 . Sori only in one side of the secondary veins

85. Venation anastomosing near the lamina margin Hemidictyum marginatum

85. Venation free

86. Lamina 2-4-pinnate at base

87. Rachis with proliferous buds Asplenium radicans

87. Rachis without proliferous buds

88. Plants epiphyte; fronds pendulous; stem with linear scale; green lamina Asplenium scandicinum

88. Plants terrestrial; fronds erects; stem with lanceolate scale; bluish-green lamina .Asplenium pseudonitidum

86. Lamina 1-pinnate at base

89. Stem long-creeping with coloration conspicuously green Hymenasplenium triquetrum

89. Stem erect or decumbent with coloration little evident

90. Lamina margin entire to weakly crenate Asplenium oligophyllum

90. Lamina margin weakly or deeply incised (serrate)

91. Apex of the segments mucronate

92. Petiole green and glabrous Asplenium mucronatum

92. Petiole greyish with linear-lanceolate scales Asplenium mullerianum

91. Apex of the segments not mucronate

93. Pinnae acroscopically with an auricle overlapping the rachis

Asplenium auriculatum

93. Pinnae acroscopically without auricle or, if it is present, not overlapping the rachis

94. Proximal pinnae gradually reduced ( $1 / 2$ the length of medial or smaller)

95. Fronds erect; petiole long about $1 / 3$ of the lamina length

Asplenium claussenii

95. Fronds pendulous; petiole short about $1 / 10$ of the lamina length Asplenium pteropus

94. Proximal pinnae not gradually reduced or only slightly reduced

96. Apex of the lamina without proliferous buds Asplenium raddianum

96. Apex of the lamina with proliferous buds Asplenium kunzeanum 
65. Sori not linear

97. Sori acrostichoid

98. Venation anastomosing

99. Fronds 2-4 m long; spores trilete Acrostichum danaeifolium

99. Fronds smaller than $2 \mathrm{~m}$ long; spores monolete

100. Pinnae articulate to the rachis

Mickelia scandens

100. Pinnae not articulate to the rachis

101. Sterile pinna with basiscopic side excavate; veins joined by a submarginal vein (inconspicuous in dry material) Olfersia cervina

101. Sterile pinna with basiscopic and acroscopic sides conform; veins conspicuously areolate Bolbitis serratifolia

98. Venation free

102. Lamina 1-pinnate; pinnae entire articulate with the rachis

Lomariopsis marginata

102. Lamina 1-3-pinnate-pinnatisect; pinnae pinnate or more divided not articulate to the rachis

103. Stem scales with entire margins and recurved base; proximal pinnae 3-pinnate-pinnatisect Polybotrya cylindrica

103. Stem scales with eroded margins to slightly denticulate and base not recurved; proximal pinnae pinnate-pinnatisect Polybotrya semipinnata

97. Sori not acrostichoid

104. Sporangia gathered in synangium or in spikes

105. Plants terrestrial; rhizome fleshy and protected by amilaceous stipules; sporangia in synangium Danaea geniculata

105. Plants climbing; rhizome long-creeping without stipules; sporangia in spikes Lygodium volubile

104. Sporangia not gathered in synangium or spikes

106. Pair of fertile pinnae modified in spike; sporangia pyriform with apical annulus

107. Terminal pinna conform; equilateral pinna base; anastomosing veins Anemia phyllitidis

107. Terminal pinna pinnatifid; pinna base not equilateral; free veins Anemia mandioccana

106. Pair of fertile pinnae not modified in spike; sporangium globose with oblique, lateral or vertical annulus 108. Sporangium with oblique annulus

109. Fronds 3-4-pinnate-pinnatifid; rhizome erect and stout with golden hairs, 4-10 mm long; plants subarborescent Lophosoria quadripinnata

109. Fronds dichotomously divided (pinnae furcate); rhizome creeping with scales or hairs (up to $4 \mathrm{~mm}$ long); plants herbaceous

110. Buds protected by hairs; veins 2-4-furcate

Gleichenella pectinata

110. Buds protected by scales; veins one time furcate

111. Axes scales patent; segments $>1.5 \mathrm{~cm}$ long Sticherus nigropaleaceus

111. Axes scales appressed; segments $<1.5 \mathrm{~cm}$ long Sticherus bifidus

108. Sporangium pedicelate with annulus vertical interrupted by the pedicel

112. Spores with chlorophyll

113. Petiole and lamina glabrous, lacking brown setae Cochlidium serrulatum

113. Petiole and lamina conspicuous with brown setae and hyaline hairs

114. Stem scales clathrate Melpomene pilosissima

114. Stem scales not clathrate 
115. Segments veins simple or only one in the acroscopic branch .....

. Moranopteris achilleifolia

112. Spores without chlorophyll

116. Sporangia sparse on the abaxial lamina surface protected by whitish wax

Ptyrogramma calomelanos

116. Sporangium gathered in defined sori and the lamina lacking white wax

117. Lamina 2-pinnate; sori oblong

118. Fronds large (1-2 m long); sori abaxial .....

Didymochlaena truncatula

118. Fronds small (not exceed $1 \mathrm{~m}$ long); sori marginal Adiantum windischii

117. Lamina 1-pinnate, sori round, not elongate

119. Pinnae articulate to the rachis

120. Indusium reniform; lamina glabrous abaxially ..phrolepis cordifolia

120. Indusium orbicular; lamina with scales and/or hairs abaxially, the scales fimbriate, the hairs catenate

121. Costa adaxially with hairs catenate

Nephrolepis biserrata

121. Costa adaxially without hairs or with sparse scales Nephrolepis rivularis

119. Pinnae not articulate to the rachis

122. Costa not sulcate adaxially

123. Veins anastomosing Tectaria incisa

123. Veins free

124. Petiole with two vascular bundles at base; lamina pubescent, the hairs acicular and septate torresiana .. Macrothelypteris

124. Petiole with more than vascular bundles at base; lamina pubescent, the hairs catenate or clavate

125. Rachis adaxially with two prominent edges; clavate hairs on the adaxial surface of the axis Lastreopsis amplissima

125. Rachis adaxially lacking prominent edges, catenate hairs on the adaxial axis

126. Rachis and costae lacking scales Ctenitis deflexa

126. Rachis and costae with clathrate scales

Ctenitis submarginalis

122. Costa sulcate adaxially

127. Veins clavate at apex, ending before the lamina margin; lamina with internal glands punctuated and translucent

128. Lamina 1-pinnate-pinatissect Stigmatopteris caudata

128. Lamina 1-pinnate, entire pinnae with crenate margin ..... Stigmatopteris heterocarpa

127. Veins not clavate at apex, ending at margin of the lamina; lamina without internal glands

129. Lamina 3 or 4 pinnate; indusium double (adaxially formed by the green laminar tissue and abaxially scarious and slender); spores trilete ......................................Saccoloma brasiliense

129. Lamina 2-pinnate; indusium single or absent; spores monolete 
130. Lamina 1-pinnate; venation anastomosing, meniscioid

131. Pedicel of the sporangium with setiform hairs Thelypteris longifolia

131. Pedicel of the sporangium without setiform hairs

132. Lamina glabrous abaxially; capsule of the sporangium with setiform hairs Thelypteris angustifolia

132. Lamina pubescent abaxially; capsule of the sporangium glabrous Thelypteris salzmannii

130. Lamina 1-pinnate-pinnatifid or 1-pinnate-pinnatisect; venation free or only the proximal veins of the adjacent segments anastomosing (not meniscioid)

133. Proximal pinna greater than or with the same size of the medial ones, or reduced, with proximal veins of the adjacent segments joining in the sinus or below the sinus (subg. Cyclosorus) Thelypteris interrupta

133. Proximal pinnae smaller than the medial ones, gradually or abruptly reduced, with proximal veins of the adjacent segments joining to the margin above the sinus (subg. Amauropelta)

134. Glandular hairs absent on the laminar tissue and/or veins, costa and margins Thelypteris eriosora

134. Glandular hairs present on the laminar tissue and/or veins, costa, and margins

135. Costa of the segments lacking scales abaxially; aerophores absent Thelypteris opposita

135. Costae of the segments with inconspicuous fimbriate scales; aerophores present on the base of the pinnae abaxially

Thelypteris metteniana 